\title{
MODELS FOR THE SIZE AND SHAPE OF THE EARTH'S MAGNETOPAUSE AND BOW SHOCK
}

\author{
J. K. Chao ${ }^{1}$, D. J. Wu ${ }^{1,2}$, C.-H. Lin ${ }^{1,6}$, Y.-H. Yang ${ }^{1}$, X.Y. Wang ${ }^{1,3}$, M. Kessel ${ }^{4}$, S.H. Chen ${ }^{4}$, and R.P. Lepping ${ }^{5}$ \\ ${ }^{1}$ Institute of Space Science, National Central University, Chung-li, Taiwan 32001 \\ ${ }^{2}$ Purple Mountain Observatory, Academia Sinica, Nanjing 210008 \\ ${ }^{3}$ Center for Space Science and Applied Research, Academia Sinica, Beijing 100080 \\ ${ }^{4}$ NSSDC, GSFC, NASA, Greenbelt, MD 20771 \\ ${ }^{5}$ Planetary Magnetosphere Branch, Laboratory for Extraterrestrial Physics GSFC/NASA, Greenbelt, MD 20771 \\ ${ }^{6}$ Department of Electrical Engineering , Nan-Jeon Institute of Technology, Yen-Shui, Taiwan 737
}

\begin{abstract}
New models for the size and shape of the Earth's magnetopause and bow shock are derived, based on a criterion for selecting the crossing events and their corresponding up-stream solar wind parameters. In this study, we emphasize the importance of accurate interplanetary parameters for predicting the size and shape of the magnetopause and bow shock. The time lag of the solar wind between the solar wind monitor and the location of crossings is carefully considered, ensuring more reliable up-stream solar wind parameters. With this database new functional forms for the magnetopause and bow shock surfaces are deduced. In this paper, we briefly present the preliminary results. For a given up-stream solar wind dynamic pressure $D_{p}$, an IMF north-south component $B_{z}$, a solar wind $\beta$ and a magnetosonic Mach number $M_{m s}$, the parameters that describe the magnetopause and bow shock surfaces $r_{0}$ and $\alpha$ can be expressed in terms of a set of coefficients determined with a multi-parameter fitting. Applications of these models to extreme solar wind conditions are demonstrated. For convenience, we have assumed that $r_{0}, B_{z}$ and $D_{p}$ retain their units, except in equations where they are normalized by $1 R_{E}$ (Earth radius), $1 \mathrm{nT}$ and $1 \mathrm{nPa}$, respectively.
\end{abstract}

\section{INTRODUCTION}

The magnetopause (MP) and bow shock (BS) play an important role in protecting the Earth from the harmful effects of the solar wind. Generally speaking, subsolar points of the MP and BS are located at about 10 and $15 R_{E}$, respectively, from the Earth under normal solar wind conditions. Chapman and Ferraro [1931] first suggested the existence of an MP boundary. Subsequently, many MP models have been proposed [Fairfield, 1971; Holzer and Slavin, 1978; Sibeck et al., 1991; Petrinec et al., 1991; Petrinec and Russell, 1993, 1996; Roelof and Sibeck, 1993; Shue at al., 1997, 1998; Kuznetsov and Suvorova, 1998; Kawano et al., 1999]. Most MP models use either a general equation of an ellipsoid with two parameters (the eccentricity and standoff distance) or a general quadratic equation. Cairns et al. [1995] discussed the limitations of using an elliptic equation. Recently, Shue et al. [1997] presented a new function to fit the size and shape of the MP:

$$
r=r_{0}\left(\frac{2}{1+\cos \theta}\right)^{\alpha}
$$

where $r_{0}$ and $\alpha$ are the standoff distance and the level of tail flaring, respectively, and $(r, \theta)$ are polar coordinates in the ecliptic plane with the origin at the Earth's center and the axis along the Sun-Earth line. The model is derived from crossings near the ecliptic plane. Thus, the model may not be suitable for use with high-latitude crossings. For high-latitude crossings, the model of Boardsen et al. [2000] can be applied. In the present study, we use the same functional form as Shue et al. [1997]. However, the database differs from that of their model by using a more accurate time shift of the solar wind propagating from IMP 8 (or ISEE 3) to the Earth. So that the database can be used for extreme solar wind conditions, the MP crossings by geosynchronous satellites are included. We will discuss the possible bias due to the satellite's particular orbits and how it can be minimized in our model. 
The study of the large-scale size and shape of the Earth's BS has a long history [see for example Beard, 1960, 1962; Midgley and Davis, 1962; Spreiter et al., 1966; Dryer and Heckman, 1967; Gosling et al., 1967; Russell et al., 1968; Behannon, 1968; Binsack and Vasyliunas, 1968; Egidi et al., 1970; Fairfield, 1971; Formisano et al., 1971, 1973; Formisano et al, 1973; Spreiter and Rizzi, 1974; Villante, 1976; Russell, 1985; Formisano, 1979; Slavin and Holzer, 1981; Slavin et al., 1984; Greenstadt et al., 1990; Farris et al., 1991; Nemecek and Safrankova, 1991; Cairns and Grabbe, 1994; Farris and Russell, 1994; Cairns and Lyon,1995; Cairns et al., 1995, 1996; Peredo et al., 1995; Lepidi et al., 1996; Russell and Petrinec, 1996; Slavin et al., 1996; Bennett et al., 1997, and references therein]. On the theoretical side, the studies are mainly based on numerical simulations using gas dynamic and hydromagnetic approaches [Spreiter et al., 1966; Dryer and Heckmann, 1967; Spreiter and Rizzi, 1974; Cairns and Lyon, 1995; Song et al., 1999]. Much attention has been given to the fitting and empirical modeling of the BS boundaries. The purpose of those works was to test and refine theoretical models of the solar wind flow about the magnetosphere. This boundary is readily identifiable and the observed position and shape can be used to validate theoretical predictions. In addition, the development of empirical models can meet operational needs and the contributions of such models may improve our understanding of the solar wind/magnetosphere coupling which is important for space weather studies. For our BS model we use a similar functional form to that used by Shue et al. [1997] for the MP (i.e. the same form as Eq. 1). The parameters $r_{0}$ and $\alpha$ that describe the BS surface are functions of $B_{z}, D_{p}, \beta$ and $M_{m s}$, determined with a multi-parameter fitting. In the following sections we describe our derived models of the MP and BS.

\section{A MAGNETOPAUSE MODEL}

We have improved the database of MP crossings used by Shue et al. [1997, 1998] based on the following considerations. In the old database, the time shift of solar wind propagating from IMP 8 (or ISEE 3) to the Earth is assumed to be constant for simplicity (10 minutes for IMP 8 and 50 minutes for ISEE 3 ). However, the speed of the solar wind can change substantially in a 10-minute (or 50-minute) interval and hence the transient time can vary by a large amount. This transient effect appears to be very important especially for those MP crossings that occur near the Earth. Since these crossings are associated almost exclusively with sudden changes in the solar wind speed and density, using only a default time delay would lead to solar wind conditions different from the actual enhancements. Therefore, we use the actual satellite positions and the measured solar wind speed to estimate a more accurate time shift for the database. In the flank region of the magnetosphere, the BS is so weak that it cannot be detected easily. Thus, the data from IMP 8 could have been taken incorrectly as the solar wind values when the satellite was in fact in the magnetosheath. Such a situation is eliminated in the new database.

Finally, 552 crossings have been selected. The ranges of the solar wind parameters are $-15<B_{z}<15 \mathrm{nT}$ and $0.4<D_{p}<9$ $\mathrm{nPa}$. All crossings have been corrected for aberration in the GSM coordinate system. Our model has the same functional form as that of Shue et al. [1997], but the way in which $r_{0}$ and $\alpha$ depend on $B_{z}$ and $D_{p}$ is different. The predicted errors in our model are approximately 10\% less than those in the Shue et al. [1998] model. In order to distinguish magnetopause responses under normal and extreme solar-wind conditions, the dependence of $r_{0}$ is derived separately. Those observed crossings with $r>6.7 R_{E}$ and $r \leq 6.7 R_{E}$ are used to derive a relationship for normal and extreme solar-wind conditions, respectively. Then these relationships are used as the models for normal and extreme solar-wind conditions with $r_{0} \geq 7.0$ $R_{E}$ and $r_{0} \leq 6.4 R_{E}$, respectively. The reason for choosing $r_{0} \geq 7.0 R_{E}$ as the boundary of normal solar wind conditions is somewhat arbitrary. Since all three models under consideration can predict the magnetopause crossings very well for $r_{0} \geq$ $7.0 R_{E}$, we use one model to describe this region. Because the $r_{0}$ vs $D_{p}$ dependence is very different in the regions $r>6.7$ $R_{E}$ and $r \leq 6.7 R_{E}$, we choose $r_{0} \leq 6.4 R_{E}$ as the boundary for extreme solar wind conditions. This choice is obtained through an iterative procedure, described in the latter part of this section. A continuous and smooth variation of $r_{0}$ is required between 6.4 and 7.0 $R_{E}$. It is found that if we assume $\ln \left(r_{0}\right)$ vs $D_{p}$ is linear in the region $6.7<r_{0}<7.0 R_{E}$, but $r_{0}$ vs $D_{p}$ is linear in the region $6.4<r_{0} \leq 6.7 R_{E}$, then we have a continuous and smooth transition such that both $r_{0}$ and $\mathrm{d} r_{0} / \mathrm{d} D_{p}$ are continuous functions of $D_{p}$, while $\mathrm{d} r_{0} / \mathrm{d} D_{p}$ is always negative from normal to extreme conditions. The total errors from the best fit with interpolation are found to remain the same as that without interpolation. Then, our model is obtained as follows:

$$
\begin{gathered}
r 0=\left\{\begin{array}{ccc}
\left(a_{1}\right)\left(D_{p}\right)^{-1 / a_{4}} & \text { for } & B_{z} \geq 0 \\
\left(a_{1}+a_{2} B_{z}\right)\left(D_{p}\right)^{-1 / a_{4}} & \text { for } & -8 \leq B_{z}<0 \\
\left(a_{1}+8 a_{3}-8 a_{2}+a_{3} B_{z}\right)\left(D_{p}\right)^{-1 / a_{4}} & \text { for } & B_{z}<-8
\end{array}\right. \\
\alpha=\left(a_{5}+a_{6} B_{z}\right)\left(1+a_{7} D_{p}\right)
\end{gathered}
$$

In deriving our model, the following three assumptions have been made. First, the functional forms in terms of $D_{p}$ and $B_{z}$ for normal and extreme conditions are assumed to be the same, except that the coefficients are different. Second, the subsolar distances $r_{0}$ for $B_{z}>0$ depend only on $D_{p}$ with a power index equal to $-1 / a_{4}$, where $a_{4}$ can differ between normal and extreme conditions. Third, the coefficients $a_{1}, a_{5}, a_{6}$ and $a_{7}$ do not change between normal and extreme conditions. With these assumptions, we derive the coefficients $a_{1}=11.646, a_{2}=0.216, a_{3}=0.122, a_{4}=6.215, a_{5}=0.578, a_{6}=-0.009$ and $a_{7}=0.012$ for the normal solar wind conditions (i.e. $r_{0} \geq 7.0 R_{E}$ ). The standard deviation (SD) from the best fit for the 
normal solar wind conditions is $1.14 R_{E}$. It is also found that the coefficients for extreme conditions (i.e. $\left.r_{0}<6.4 R_{E}\right)$ are $a_{1}=$ 11.646, $a_{2}=0.169, a_{3}=0.158, a_{4}=6.800, a_{5}=0.578, a_{6}=-0.009$ and $a_{7}=0.012$, with SD $=0.55 R_{E}$ from the best-fit procedure. For $B_{z}>0$, the function for region $6.7<r_{0}<7.0 R_{E}$ is $\ln \left(r_{0}\right)-\ln (7)=C_{l}\left(D_{p}-C_{2}\right)$, where $C_{I}=-0.003$ and $C_{2}=$ 23.7. The function for $6.4<r_{0}<6.7 R_{E}$ is $r_{0}-6.4=C_{3}\left(D_{p}-C_{4}\right)$, where $C_{3}=-0.015$ and $C_{4}=58.6$ calculated from the first equation of Eq. (2). As for $B_{z}<0$, the coefficients $C_{1}, C_{2}, C_{3}$ and $C_{4}$ can also be calculated from the other equations in Eq. (2) which have a $B_{z}$ dependence.

In deriving the model for the extreme solar wind conditions, the MP crossings observed by geosynchronous satellites are taken into consideration. The possible bias due to the satellite's particular orbits may be minimized in deriving a model for $r_{0}=6.6$ $R_{E}$ if we use the following procedure. Figure 1 shows the predicted contour plots of $r_{0}=6.6 R_{E}$ for various MP models. The models of Shue et al. [1998] and Petrinec and Russell [1996] are shown as dashed and dotted lines, respectively. The solid line between the dashed and dotted lines is the prediction of $r_{0}=6.6 R_{E}$ from our model for the normal solar wind condition. The crosses in the figure are the interplanetary $D_{p}$ and $B_{z}$ values associated with those crossings that were observed by GOES satellites, implying that these MP have already moved inside the geosynchronous orbit. By inspecting the positions of these three contours with respect to the crosses, it is apparent that the predicted $r_{0}=6.6 R_{E}$ contours are slightly outside the boundaries of the crosses. Ideally, the contours should be as close as possible to the outer edge of the crosses.

Intuitively, a better model may be obtained if the contour $r_{0}=6.6 R_{E}$ is moved toward the boundary of these crosses. So that the model does not over-predict and a false alarm can be minimized, we need the contour of $r_{0}=6.6 R_{E}$ to be well inside the outer boundary of the crosses. Once the location of $r_{0}=6.6 R_{E}$ is determined, we anticipate that our model will cover the region $r_{0}>6.4 R_{E}$. In deriving the model for the extreme condition (i.e. $r_{0}<6.7 R_{E}$ ), we first require that the contour $r_{0}=6.6$ $R_{E}$ is as close as possible to the boundary of the crosses. Secondly, we require a smooth transition from the normal to extreme conditions of $r_{0}$ in the region $(7.0,6.4)$ in which a linear $\ln \left(r_{0}\right)$ vs $D_{p}$ relationship is assumed for the interval (7.0, 6.7), but a linear $r_{0}$ vs $D_{p}$ applies in interval $(6.7,6.4)$. This procedure requires iteration.

We have a model covering the ranges of both the normal and extreme solar wind conditions. Since there were no crossings available for $r_{0}<6.4 R_{E}$ in the derivation of our model, the extreme solar wind condition cannot be applied for regions much less than $r_{0}=6.6 R_{E}$. Hence, our prediction is not reliable in the region $r_{0}<6.4 R_{E}$. The contour $r_{0}=6.6 R_{E}$ thus obtained is shown in Figure 1 as the solid line on the far right-hand side. The prediction of our model is now quite different from that of the other two models. However, under normal solar wind conditions, all three models predict very similar values of $r_{0}$. Note that no change of the $a$ coefficients for $\alpha$ is assumed from the normal to extreme conditions

The contours of $r_{0}$ and $\alpha$ for the normal and extreme solar wind conditions are given in Figures 2(a) and 2(b). Under extreme solar wind conditions, Yang et al. [2001] have shown that our model predicts more accurate geosynchronous MP crossings, as given in Table 1, where the three parameters are defined: probability of prediction (PoP), probability of detection (PoD) and false alarm rate (FAR). These parameters quantify the forecasting capability of a model. Higher PoP and PoD values with a lower FAR imply a better forecasting model. It is evident that our model gives a better prediction.

Table 1. Comparison of prediction capabilities for three models from 1986-1992(unit: \%)

\begin{tabular}{|c|c|c|c|}
\hline Model & $\underline{\mathrm{PoP}}$ & $\underline{\text { PoD }}$ & $\underline{\text { FAR }}$ \\
\hline Yang et al. [2001] & 79 & 96 & 69 \\
\hline Shue et al. [1998] & 67 & 93 & 78 \\
\hline
\end{tabular}

Petrinec and Russell [1996]
The models of Petrinic and Russell (1996) and Chao et al. (1997) predict that a larger southward value of $B_{z}$ can push the magnetopause further closer to the Earth regardless how large the value of the negative $B_{z}$ is. On the other hand, the models of Shue et al. [1998] and Kuznetsov and Suvorova [1998] suggest that the value of the negative Bz has a limit. In addition, the sheath encounters shown in Figure 6 (pluses) of Yang et al. [2001] seem to suggest that the negative $B_{z}$ cannot further reduce the magnetopause distance when $B_{z}$ become less than a certain negative value. We describe the $B_{z}$ influence as 'saturated' [Dmitriev et al., 2001]. From an event study, we also found that the saturation can be approximated by $B_{z}=\left(-12-D_{p}\right)$ if we have $B_{z}<(-12-$ $D_{p}$ ). The parameter $\alpha$ in Eq. (1) controls the level of tail 


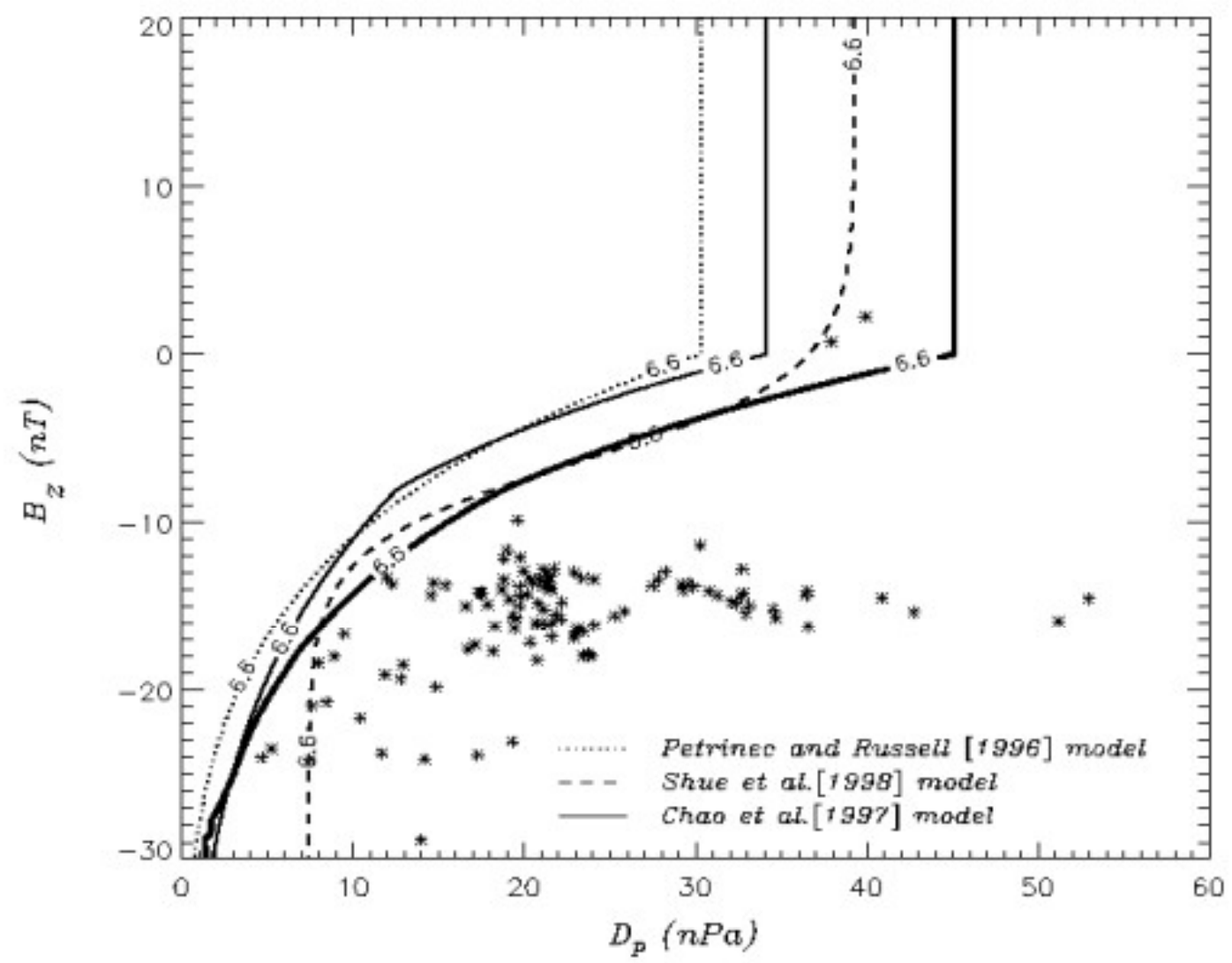

Fig.1. Predicted contour plots of $r_{0}=6.6$ Re for various magnetopause models. Models of Shue et al. [1998] and Petrinec and Russell [1996] are shown as dashed and dotted lines, respectively. The solid line between the dashed and dotted lines is the extrapolated values for $r_{0}=6.6 \mathrm{Re}$ from our model in the normal solar wind condition. The other solid line is from our present model. The cross-signs show the $D_{p}$ and $B_{z}$ values when GOES's are inside the magnetosheath.
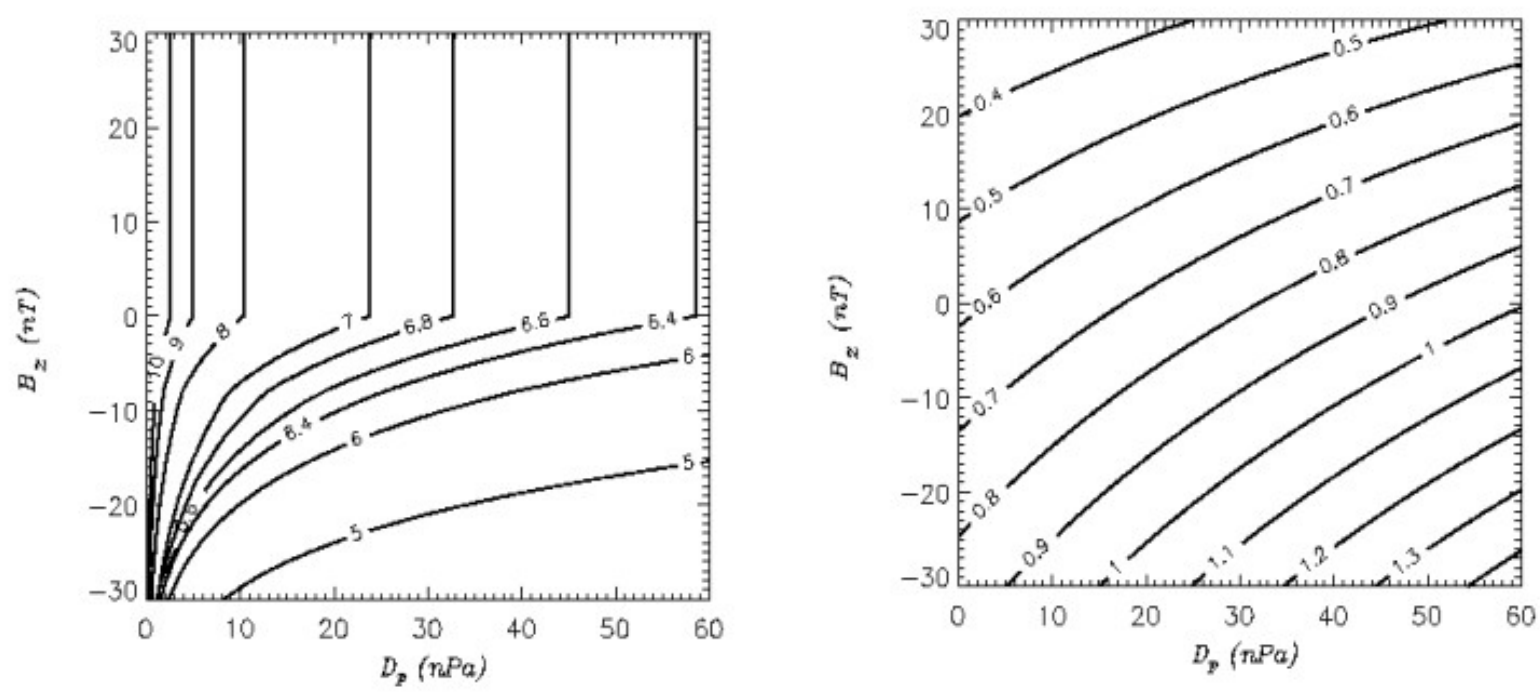

Fig. 2. Left: Contour plots for $r_{0}$ as a function of $D_{p}$ and $B_{z}$ for both normal and extreme solar wind conditions (Chao, 1997). Right: Contour plots for $\alpha$ as a function of $D_{\mathrm{p}}$ and $B_{z}$ for our new model (Chao, 1997). 
flaring of the magnetopause. However, the contour plots of $\alpha$ in Figure 2 are derived from crossings under normal solar wind conditions that may not be reasonable for large $D_{p}$. Therefore, we suggest that the dependence of $\alpha$ on $D_{p}$ should gradually diminish when $D_{p}$ approaches a large value. Thus, we assume that the coefficient $a_{7}=a_{7}{ }^{*} \exp \left(-D_{p} / 10\right)$. Eventually, these two effects will be considered in detail in future studies.

\section{A BOW SHOCK MODEL}

To develop an empirical model for the size and shape of the Earth's bow shock, the selection of the BS crossings and their associated upstream parameters are important. The solar wind parameters are not constant during the periods of the BS crossing. A sharp change in solar wind parameters often causes the crossings. The hourly average values of the solar wind are included in the BS database in some earlier work, but they may not be correct. The actual values responsible for the BS crossings can be quite different from the hourly average values. When there are sharp changes in the solar wind parameters, the BS will move from a previous equilibrium to a new one. Since the satellite's location is taken as the BS equilibrium position when the satellite passes by, the real equilibrium position of the BS is not properly obtained.

To derive a BS database, we have used high-resolution data from the ISTP-Key parameters of WIND and GEOTAIL. These BS crossings in quasi-steady conditions are chosen for our BS database. In one of the following two cases, with upstream solar wind conditions, we have selected the two BS crossings:

(1) Only one BS crossing is observed and occurs slowly.

(2) In the case of multiple crossings, only the middle one is selected to represent and weighted all crossings.

The crossings that are apparently transient events caused by sharp changes of solar wind parameters have not been selected for our database. To demonstrate the selection of quasi-steady BS and their corresponding upstream solar wind parameters, we present two examples. In Figure 3, the solar wind parameters observed by WIND (solid curves) and the magnetic fields observed by GEOTAIL (dashed curves) during 0700-1400UT, April 11, 1996 are shown. At this time, WIND is located at $\sim 80 R_{E}$ upstream from the GEOTAIL and observed solar wind velocity at $\sim 425 \mathrm{~km} / \mathrm{sec}$. The times of GEOTAIL have been properly shifted by an amount equal to solar wind travel-times from WIND to GEOTAIL.

From the top to bottom panels of Figure 3, the magnitudes of the magnetic field $B_{t}$ and its two components $B_{y}$ and $B_{z}$, together with $D_{p}$ and $M_{m s}$, are shown. By comparing the solid and the dashed curves, it is possible to identify GEOTAIL's repeated crossings of the BS. We select two crossings observed under steady-state solar wind conditions in this figure. During these two periods, the solar wind parameters were very stable, as can be seen in the figure. Therefore we suggest that the observed BS is at equilibrium for the corresponding solar wind conditions.

In our second example, multiple crossings observed by GEOTAIL are shown in Figure 4. Multiple crossings occurring with quasi-steady upstream conditions indicate that the BS is close to a steady state. We take the middle crossing as our selected event. At this time, WIND is $231.7 R_{E}$ upstream of the Earth observed at a solar wind velocity of $\sim 640 \mathrm{~km} / \mathrm{sec}$. Thus, the solar wind traveling time is approximately 38 minutes. We take this to be the time shift for GEOTAIL as shown by the dashed curves in Figure 4. Note that the first BS crossing at about 16:45 UT cannot be used for our database because the fast crossing by GEOTAIL indicates that the BS has moved out too far from the location of GEOTAIL. With this constraint, only 265 such events were selected for the period 1995-97 from GEOTAIL using WIND as the solar wind monitor. With proper weight added to the events, we finally obtained an equivalent of 1438 crossings for our fitting to derive a BS model.

The size and shape of the BS surface in aberrated polar coordinates with the origin at the Earth's center is described by a nonlinear function similar to Eq. (1) for the MP:

$$
r=r_{0}\left(\frac{1+\varepsilon}{1+\varepsilon \cos \theta}\right)^{\alpha}
$$

where $r$ is the radial distance from the Earth to the predicted BS along the Earth-satellite line, $r_{0}$ is the standoff distance of the BS, $\theta$ is the cone angle from the $x$-axis, $\varepsilon$ is a parameter similar to the eccentricity, and $\alpha$ is the level of tail flaring.

In order that our BS model is consistent with the results of the distant crossings (Bennett et al., 1997), the parameter $\varepsilon$ has been included in Eq. (3). The parameters $r_{0}$ and $\alpha$ are both functions of $B_{z}, D_{p}, \beta$ and $M_{m s}$. The BS with aberration corrected is treated to be axi-symmetric. The effect of the $y$-component of solar wind velocity $V_{y}$ is also considered in the aberration. The BS model is obtained by using a multi-parameter fitting scheme. The optimization is carried out by a chi-squared minimization of the difference between model predictions and observations. The best-fit results for the parameters $r_{0}, \alpha$ and $\varepsilon$ can be 
expressed as the following two sets of equations, depending on the sign of $B_{z}$ (Chao et al., 1999).

$$
\begin{aligned}
& r_{0}=a_{1}\left(1+a_{2} B_{z}\right)\left(1+a_{9} \beta\right)\left(1+a_{4} \frac{\left(a_{8}-1\right) M_{m s}^{2}+2}{\left(a_{8}+1\right) M_{m s}^{2}}\right) D_{p}^{-1 / a_{11}} \text { for } B_{z} \geq 0 \\
& \left.\alpha=a_{5}\left(1+a_{13} B_{z}\right)\left(1+a_{7} D_{p}\right)\left[1+a_{10} \ln (1+\beta)\right)\right]\left(1+a_{14} M_{m s}\right) \\
& r_{0}=a_{1}\left(1+a_{3} B_{z}\right)\left(1+a_{9} \beta\right)\left(1+a_{4} \frac{\left(a_{8}-1\right) M_{m s}^{2}+2}{\left(a_{8}+1\right) M_{m s}^{2}}\right) D_{p}^{-1 / a_{11}} \text { for } B_{z}<0 \\
& \alpha=a_{5}\left(1+a_{6} B_{z}\right)\left(1+a_{7} D_{p}\right)\left[1+a_{10} \ln (1+\beta)\right]\left(1+a_{14} M_{m s}\right)
\end{aligned}
$$

where $\varepsilon=a_{12}$ and the coefficients are shown below:

$$
\begin{array}{lllll}
a_{1}=11.1266 & a_{2}=0.0010 & a_{3}=-0.0005 & a_{4}=2.5966 & a_{5}=0.8182 \\
a_{6}=-0.0170 & a_{7}=-0.0122 & a_{8}=1.3007 & a_{9}=-0.0049 & a_{10}=-0.0328 \\
a_{11}=6.047 & a_{12}=1.029 & a_{13}=0.0231 & a_{14}=-0.002 &
\end{array}
$$

The errors from the best-fit procedure give a standard deviation of $1.2 R_{E}$. The functional dependences of $r_{0}$ and $\alpha$ on the parameters $D_{p}, B_{z}, \beta$ and $M_{m s}$ are given in Figure 5.

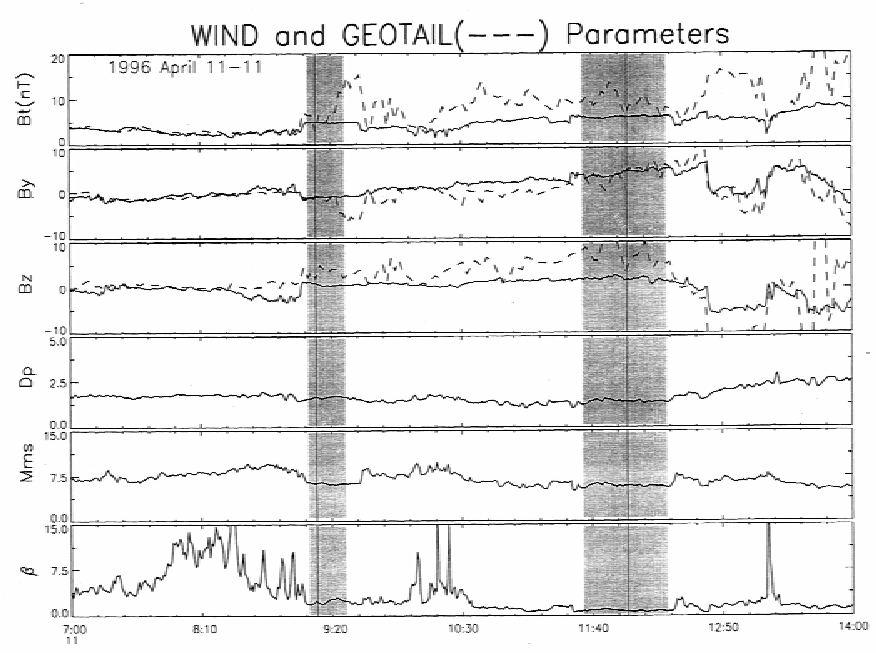

Fig. 3. Two bow shock crossings (shaded area) observed by the GEOTAIL satellite (dashed lines) at ( - 7.0, 27.5, 2.9) in GSE coordinates on April 11, 1996. The satellite passes through the shock gradually indicating that the shock is in an equilibrium position, whereas the upstream parameters (solid lines) observed by WIND during the same periods show little change. From top to bottom, the magnetic field and its two components in GSE coordinates, the solar wind dynamic pressure $D_{p}$, the magneto-sonic Mach number $M_{m s}$, and the ratio of thermal to magnetic pressure $\beta$ are shown.

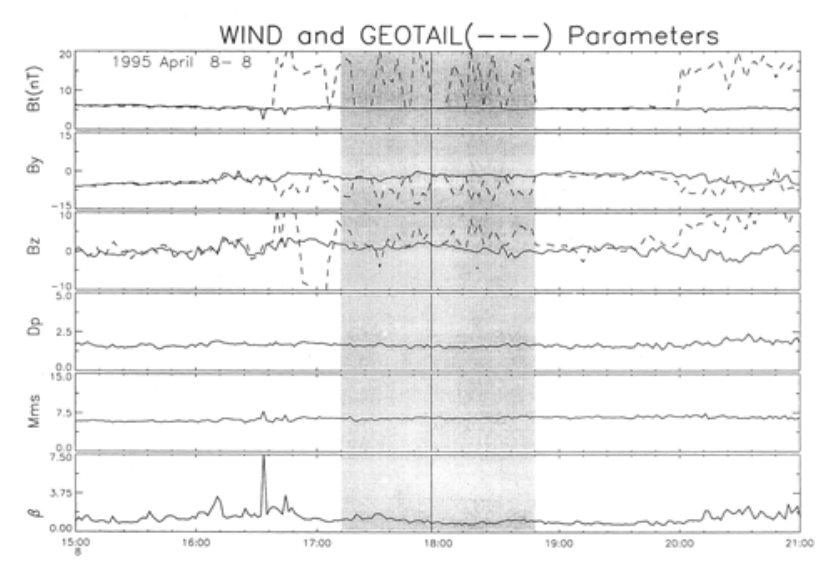

Fig. 4. Multiple bow shock crossings (shaded area) observed by GEOTAIL (dashed lines) at $(0 ., 28 .,-3.5)$ in GSE coordinates on April 8, 1995. The satellite GEOTAIL observed multiple bow shock crossings whereas the upstream parameters (solid lines) observed by WIND remain unchanged during this period indicating the bow shock is in equilibrium position. The same parameters as in Figure 1 are shown from the top to the bottom. 
Voriations of $r_{0}$ and $\alpha$ with Upstream Parameteres $B_{2}, D_{p}, M_{m g}$ and $\beta$

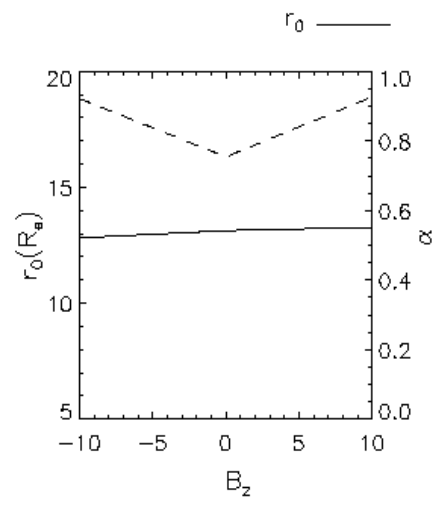

$\alpha----$
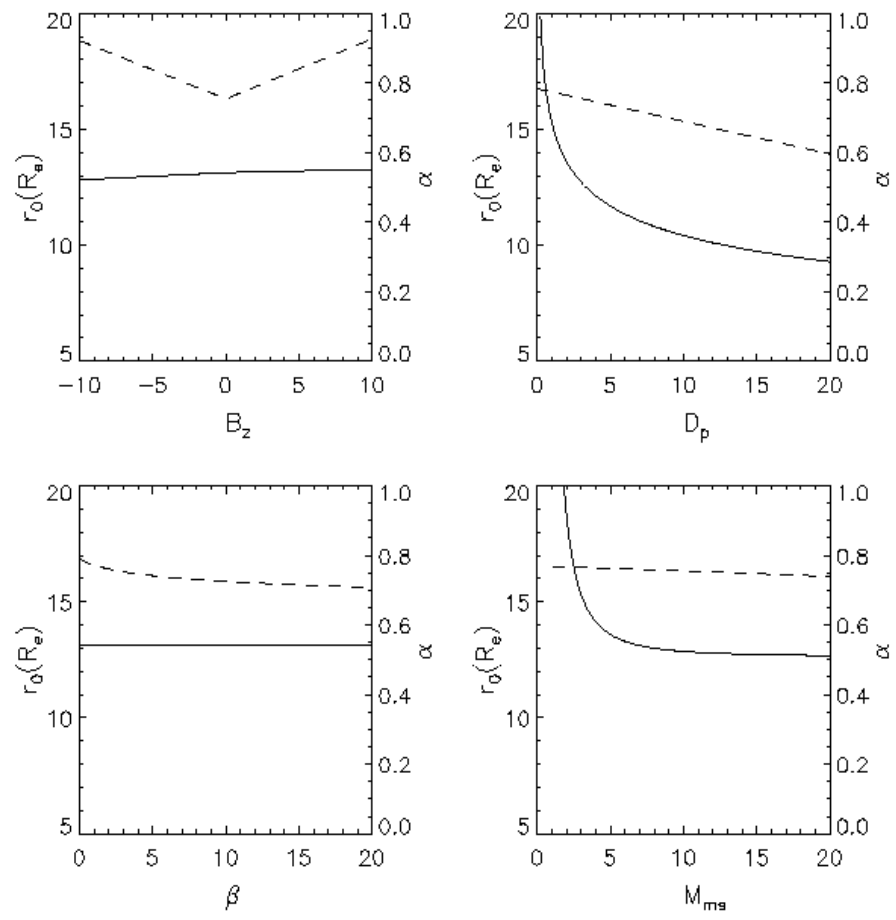

Fig. 5. Plots of $r_{0}$ (solid lines) and $\alpha$ (dashed lines) as functions of $D_{p}, B_{z}, M_{m s}$ and $\beta$ for the normal solar wind conditions, where $B_{z}=-0.35 \mathrm{nT}, D_{p}=2.48 \mathrm{nPa}$, $M_{m s}=6.96$ and $\beta=2.08$.

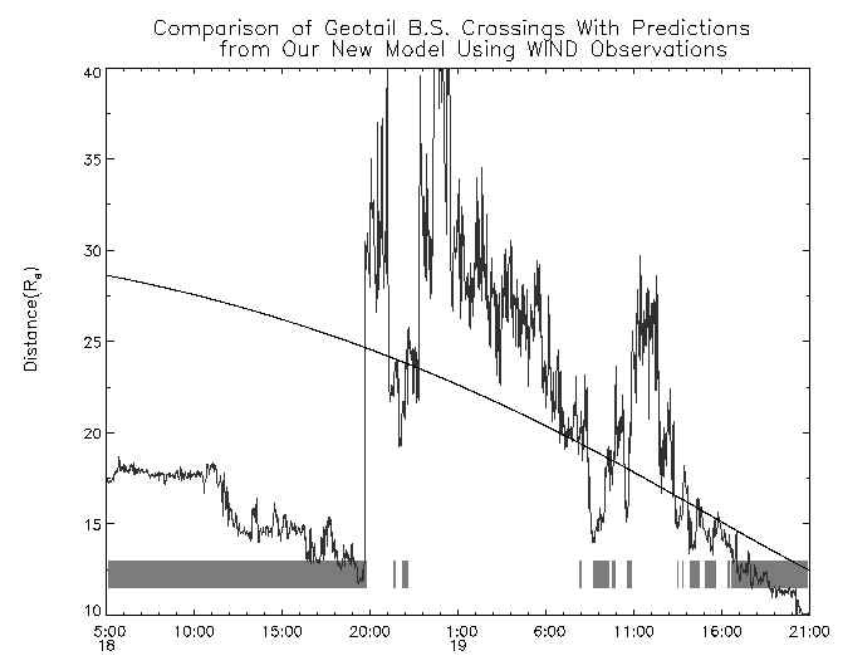

Fig. 6. Predicted distances $r$ of the bow shock (fluctuated line) and the GEOTAIL's orbit (smooth line) from the Earth's center (in units of $R_{E}$ ). The horizontal bars in the lower part of the figure indicate the actual positions of GEOTAIL in interplanetary space.
To demonstrate the capability of the model for predicting the position and shape of the Earth's BS, we give the following example. During the period October 18-20, 1995, an interplanetary magnetic cloud (IMC) was observed to pass the Earth. WIND recorded its interplanetary characteristics at $175 R_{E}$ upstream of the Earth's BS, and about 45 minutes later GEOTAIL, being near the nominal location of the dawn-side of the BS, detected multiple BS crossings. Using the data from WIND, we predicted the changes in position and shape of the BS from our semi-empirical BS model, caused by the interaction of the IMC with the magnetosphere. Figure 6 shows a comparison of the BS motion predicted by the model with GEOTAIL observations of the actual BS crossings. Both the distance of GEOTAIL from the Earth's center (i.e. corresponding to GEOTAIL's trajectory) and the distance of the BS from the Earth's center along the Earth-GEOTAIL direction, as predicted from our semi-empirical model at a time resolution of 1.5 minutes, are shown as a function of time. The transient time from WIND to GEOTAIL has been correctly shifted. Our prediction of the BS crossings is shown as the intersection points of the predicted BS distances (solid lines) and the trajectory of GEOTAIL. The horizontal bars in the lower part of the figure indicate the actual positions (or periods) of GEOTAIL being in interplanetary space; otherwise, it is in the magnetosheath. Thus, the positions when GEOTAIL changes from one region to the other represent the observed crossings by GEOTAIL. It is apparent from this figure that our predictions agree very well with observation.

\section{DISCUSSION AND SUMMARY}

In our derivation of the models for both the MP and BS, we have assumed that the subsolar distances are proportional to $D_{p}$ to a power of $-1 / a$, as in Eqs (2) and (4) where in theory $a=6.0$ for an ideal dipole magnetosphere. The empirically determined $a$ values for both the MP and BS are close to 6.0, indicating our assumed functional form of $D_{p}$ is close to the ideal dipole result. For the case of the MP, $r_{0}$ and $\alpha$ are functions only of $D_{p}$ and $B_{z}$. Since the observation of a southward $B_{z}$ related to inward motion of the MP has long been recognized, magnetic reconnection may play a role in the physics of this inward motion. It is possible that the $V_{x} B_{z}$ term is more important than $B_{z}$ for controlling this inward motion. However, our preliminary study shows that using $V_{x}$ $B_{z}$ instead of $B_{z}$ increases the error in the fitting. Thus, we have continued to use $B_{z}$ as a control parameter in this study. According to Eq. (2), the subsolar position of the MP is independent of $B_{z}$ when it is northward and more strongly depends on the southward $B_{z}$ (i.e. $a_{3}$ is not much smaller than 1). Since most of the crossings of the MP are on the dayside, the parameter $\alpha$ controlling the flaring of the MP for the tail part may not be statistically significant. More tests for the distant tail crossings are needed to verify the applicability of this model to the tail region of the MP. The subsolar distance and the flaring of the BS are controlled by the four variables $D_{p}, B_{z}, \beta$ and $M_{m s}$. 
We have also noticed that the subsolar distance $r_{0}$ of BS is weakly dependent on $B_{z}$, whereas for the MP the $B_{z}$ dependence is stronger. The model has been tested successfully both for normal and for extreme solar wind conditions when the solar wind number density and Mach number can drop to much smaller than normal values [Lin et al., 2000].

In summary, we have derived new models for the size and shape of the Earth's MP and BS, based on a criterion for the selection of crossing events and their corresponding up-stream solar wind parameters. Our work emphasizes the importance of accurate interplanetary parameters in making predictions. Although these models are derived using crossings under normal solar wind condition, they have also been applied to extreme solar wind conditions with reasonable success. During the coming solar maximum, more events under extreme solar wind conditions can be used to test the models

\section{ACKNOWLEDGEMENTS}

This work was supported by grant NSC 89-2111-M-008-070 to the National Central University. The authors thank NASA/NSSDC and NOAA/NGDC for data used in this study. JKC thanks A. V. Dmitriev for useful comments.

\section{REFERENCES}

Beard, D. B., The interaction of the terrestrial magnetic field with the solar corpuscular radiation, J. Geophys. Res., 65 , 3559, 1960.

Beard, D. B., Interaction of the terrestrial magnetic field with the solar corpuscular radiation, 2, Second-order approximation, J. Geophys. Res., 67, 477, 1962.

Behanon, K. W., Mapping of the Earth's bow shock and magnetic tail by Explorer 33, J. Geophys. Res., 73, $907,1968$.

Bennett L., M. G. Kivelson, K. K. Khurana, L. A. Frank, and W. R. Paterson, A model of the earth's distant bow shock, J. Geophys. Res., 102, 26927, 1997.

Binsak, J. H., and V. M. Vasyliunas, Simultaneous IMP 2 and OGO 1 observations of bow shock compression, J. Geophys. Res., 73, 429, 1968.

Boardsen, S. A., T. E. Eastman, T. Sotirelis, and J. L. Green, An empirical model of the high-latitude magnetopause, J. Geophys. Res., 105, 23193-23220, 2000.

Cairns, I. H., and C. L. Grabbe, Towards an MHD theory for the standoff distance of Earth's bow shock, Geophys. Res. Lett., $21,2781,1994$.

Cairns, I. H., and J. G. Lyon, MHD simulations of Earth's bow shock at low Mach numbers: Standoff distances, J. Geophys. Res., 100, 17173, 1995.

Cairns, I. H., D. H. Fairfield, R. R. Anderson, V. E. H. Carlton, K. I. Paularena, and A. J. Lazarus, Unusual locations of: Earth's bow shock on September 24-25, 1987: Mach number effects, J. Geophys. Res., 100, 47, 1995.

Cairns, I. H., D. H. Fairfield, R. R. Anderson, K. I. Paularena, and A. J. Lazarus, Reply to comment on 'Unusual locations of Earth's bow shock on September 24-25, 1987: Mach number effects," J. Geophys. Res., 101, 7679, 1996.

Chao, J. K., Importance of accurate interplanetary $B_{z}$ and $D_{p}$ for the prediction of the size and shape of Earth's magnetosphere, AGU Fall Meeting, San Francisco, CA, F592, 1997, A supplement to Eos, Transactions, AGU, vol. 78, No. 78, No. 46, Nov. 18, F592, 1997

Chao, J. K., C.-H. Lin, D. J. Wu, M. Kessel, S. H. Chen, and R. Lepping, A model for the size and shape of the Earth's bow shock, EOS, Transactions, AGU 1999 Fall Meeting, Vol. 80, No. 46, F897, 1999.

Chapman, S., and V. C. A. Ferraro, A new theory of magnetic storm, I, The initial phase, J. Geophys. Res., 36, 77, 1931.

Dmitriev, A. V., J. K. Chao, and Y.-H. Yang, Saturation of the Bz-influence for the geosynchronous magnetopause crossings, Proceedings of the Seventh Conference on Atmospheric Science in Taiwan, September 25-27, 2001

Dryer, M., and G. R. Heckman, On the hypersonic analogue as applied to planetary interaction with the solar plasma, Planet. Space Sci., 15, 515, 1967.

Egidi, A., V. Formisano, F. Palmiotto, P. Saraceno, and C. Moreno, Solar wind and location of shock front and magnetopause at the 1969 solar maximum, J. Geophys. Res., 75, 6999, 1970.

Fairfield, D. H., Average and unusual locations of the Earth's magnetopause and bow shock, J. Geophys. Res., 76, 6700, 1971.

Farris, M. H., and C. T. Russell, Determining the standoff distance of the bow shock: Mach number dependence and use of models, J. Geophys. Res., 99, 17681, 1994.

Farris, M. H., S. M. Petrinec, and C. T. Russell, The thickness of the magnetosheath: Constraints on the polytropic index, Geophys. Res. Lett., 18, 1821, 1991.

Formisano, V., Orientation and shape of the Earth's bow shock in three dimensions, Planet. Space Sci., $27,1151,1979$.

Formisano, V., P. C. Hedgecock, G. Moreno, J. Sear,and D. Bollea, Observations of Earth's bow shock for low Mach numbers, Planet. Space Sci., 19, 1519, 1971.

Formisano, V., P. C. Hedgecock, G. Moreno, F. Palmiotto, and J. K. Chao, Solar wind interactions with the Earth's magnetic 
field, 2, Magnetohydrodynamic bow shock, J. Geophys. Res., 78, 3731, 1973.

Gosling, J. T., J. R. Asbridge, S. J. Bame, and I. B. Strong, Vela 2 measurements of the magnetopause and bow shock positions, J. Gecpys. Res., 72, 101, 1967.

Greenstadt, E. W., D. P. Traver, F. V. Coroniti, E. J. Smith, and J. A. Slavin, Observations of the flank of Earth's bow shock to $-110 \mathrm{R}_{\mathrm{E}}$ by ISEE3/ICE3, Geophys. Res. Lett., 17, 753, 1990.

Holzer, R. E., and J. A. Slavin, Magnetic flux transfer associated with expansions and contractions of the dayside magnetosphere, J. Geophys. Res., 83, 3831, 1978.

Kawano, H., S. M. Petrinec, C. T. Russell, and T. Higuchi, Magnetopause shape determinations from measured position and estimated flaring angle, J. Geophys. Res., 104, 247, 1999.

Kuznetsov, S. N., and A.V. Suvorova, An empirical model of the magnetopause for broad ranges of solar wind pressure and $B_{z}$ IMF, in Polar Cap Boundary Phenomena, edited by J. Moen et al., p. 51, Kluwer Acad., Norwell, Mass., 1998.

Lepidi S., Villante U., Lazarus A. J., Szabo A., and Paularena K., Observations of bow shock motion during times of variable solar conditions, J. Geophys. Res., 101, 11107, 1996.

Lin, C.-H., J. K Chao, Y.-H. Yang, and X. Y. Wang, Predictions of the Earth's Bow Shock Crossings on May 11-12, 1999, COSPAR Colloquium, Space Weather Study Using Multi-point Techniques, September 27-29, 2000.

Midgley, J. E. and L. Davis Jr., Computation of the bounding surface of a dipole field in a plasma by a moment technique, J. Geophys. Res., 67, 499, 1962.

Nemecek, Z. and J. Safrankova,The Earth's shock and magnetopause position as a result of solar wind - magnetosphere interaction, J. of Atmospheric and Terr. Phys., 53, 1049, 1991.

Peredo, M., J. A. Slavin, E. Mazur, and S. A. Curtis, Three-dimensional position and shape of the bow shock and their variation with Alfvenic, sonic and magnetosonic Mach numbers and interplanetary magnetic field orientation, J. Geophys. Res., 100, 7907, 1995.

Petrinec, S. M., and C. T. Russell, An empirical model of the size and shape of the near-Earth magnetotail, Geophys. Res. Lett., 20, 2695, 1993.

Petrinec, S. M., and C. T. Russell, Near-Earth magnetotail shape and size as determined from the magnetopause flaring angle, J. Geophys. Res., 101, 137, 1996.

Petrinec, S. M., P. Song, and C. T. Russell, Solar cycle variations in the size and shape of the magnetopause, J. Geophys. Res., 96, 7893, 1991.

Roelof, E. C., and D. G. Sibeck, Magnetopause shape as a bivariate function of interplanetary magnetic field $B_{z}$ and solar wind dynamic pressure, J. Geophys. Res., 98, 21,421, 1993.

Russell, C. T., Planetary Bow Shocks, in Collisionless Shocks in the Heliosphere: A Tutorial Review, Geo. Mono. Ser.,35, edited by B. T Tsurutani and R. G. Stone, pp. 109-130, AGU, Washington, D.C., 1985.

Russell, C. T., and S. M. Petrinec, Comment on "Unusual locations of Earth's bow shock on September 24-25, 1987: Mach number effects", J. Geophys. Res., 101, 7677, 1996.

Russell, C. T., J. V. Olson, R. E. Holzer, and E. J. Smith, OGO 3 search coil magnetometer data correlated with the reported crossing of the magnetopause at 6.6 RE by ATS I, J. Geophys. Res., 73, 5769, 1968.

Shue J. H., J. K. Chao, H. C. Fu, C. T. Russell, P. Song, K. K. Khurana, and Singer H. J., A new functional form to study the solar wind control of the magnetopause size and shape, J. Geophys. Res., 102, 9497, 1997.

Shue J.-H., P. Song, C. T. Russell, J. T. Steinberg, J. K. Chao, G. Zastenker, O. L. Vaisberg, S. Kokubun, H. J. Singer, T. R. Detman, and H. Kawano, Magnetopause location under extreme solar wind conditions, J. Geophys. Res., 103, 17,691, 1998.

Sibeck, D. G., R. E. Lopez, and E. C. Roelof, Solar wind control of the magnetopause shape, location, and motion, J. Geophys. Res., 96, 5489, 1991.

Slavin J., A. Szabo, M. Peredo, R. P. Lepping, R. J. Fitzenreiter, K. W. Ogilvie, C. J. Owen, and J. T. Steinberg, Near-simultaneous bow shock crossings by WIND and IMP 8 on December 1, 1994, Geophys. Res. Lett., 23, 1207, 1996.

Slavin, J. A., and R. E. Holzer, Solar wind flow about the terrestrial planets, 1, Modeling bow shock position and shape, J. Geophys. Res., 86, 11,401, 1981.

Slavin, J. A., R. E. Holzer, J. R. Spreiter, and S. S. Stahara, Planetary Mach cones: Theory and observation, J. Geophys. Res., $89,2,708,1984$.

Song, P., C. T. Russell, T. I. Gombosi, I. R. Spreiter, S. S. Stahara, and X. X. Zhang, On the processes in the terrestril magnetosheath : 1. Scheme development, J. Geophys. Res., 104, 22345-22355, 1999.

Spreiter, J. R., A. L. Summers, and A. Y. Alksne, Hydromagnetic Flow Around the magnetosphere, Planet. Space Sci., 14, 223, 1966.

Spreiter, J. R., and A. W. Rizzi, Aligned magnetohydrodynamic solution for solar wind flow past the earth's magnetosphere, Acta Astronaut, 1, 15, 1974.

Villante, U., Evidence for a bow shock at $\sim 400 \mathrm{R}_{\mathrm{E}}$ : Pioneer 7, J. Geophys. Res., 81, 1441, 1976.

Yang, Y.-H., J. K. Chao, C.-H. Lin, J.-H. Shue, X.-Y. Wang, P. Song, C. T. Russell, R. P. Lepping and A. J. Lazarus, Comparison of three magnetopause prediction models under extreme solar-wind conditions, J. Geophys. Res., (in press), 2001. 